\title{
Il federalismo in sanità: opportunità e rischi tra finanziamento, equità ed assistenza
}

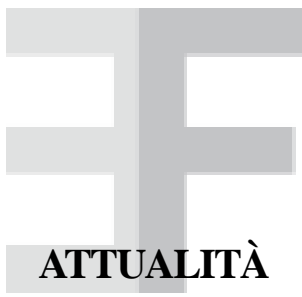

Fabrizio Gianfrate* $\$$

\begin{abstract}
Federalism in Italy arised beginning '90, pushed bipartisanly from establishment in order to contrast the succesfull poll of northern league party. Being about $80 \%$ of the regions budget for healthcare, federalism had its hard impact on it. Regions can manage autonomously their healthcare organization, adding to the State financial resources own money earning from other their services or coming from local taxes, on condition that they provide the State LEA (Essential Level of Assistance). That means a high level of responsabilization for local government for reaching a higher level of efficiency in allocation of resources and organizational models of production and erogation of healthcare, beside a better ability to catch local healthcare demand. Even if now the sharing of State financial resources are agreed between all regions and Government, it's not clear for the future wheter each region will have to procure its own finance, introducing many problems for those southern regions unable to substain theirselves. It has still to be defined wheter and how reach regions will have to transfer part of their resources to the poor ones. That means a risk of iniquity of level in healthcare provision among regions of the same country, generating possible social conflict. Therefore federalism now has from a hand the opportunity to better tail healthcare on each local demand and, on the other hand, it risks to introduce social disparity and conflicts.
\end{abstract}

Keywords: federalism in healthcare management, healthcare economics, pharmaceutical economics Farmeconomia e percorsi terapeutici 2005; 6 (3): 207-214

\section{INTRODUZIONE}

Inserite nella complessiva turbolenza delle democrazie industriali avanzate e dei loro sistemi di welfare, le problematiche del Sevizio Sanitario Nazionale italiano hanno profondi risvolti tanto economici, che sociali, tanto politici che istituzionali. La fase storica che sta attraversando adesso la sanità italiana è particolarmente delicata per i processi di riforma ispirati al federalismo che, in atto da qualche anno, ne stanno modificando sostanzialmente i diversi aspetti organizzativi, gestionali e finanziari [1].

Appare ormai chiaro il contesto di percepita perdita progressiva delle protezioni sociali dettata da un quadro macro-economico apparentemente impossibilitato a sostenere i modelli di welfare del passato. A meno di aumentare le risorse pubbliche incrementando la pressione fiscale, atto politicamente sempre difficile da compiersi e che comunque rischia di introdurre ripercussioni negative sui consumi delle famiglie e sugli investimenti delle imprese. L'alternativa è privatizzare la spesa per alcuni dei servizi spostandone l'onere economico direttamente sul paziente, rischiando però così di introdurre eccessive iniquità per reddito nell'accesso alle prestazioni. In questo con- testo appare necessario riflettere su quale sia o debba essere il diritto di soddisfazione dei bisogni sanitari da parte dello Stato. A tale proposito il ruolo di rappresentanza e di interpretazione delle comunità locali diventa cruciale, amplificando le capacità di focalizzazione e di adattamento dinamico alle esigenze della rispettiva collettività, aumentando il potenziale di efficacia e di efficienza del sistema di tutela e cura della salute, così ritagliato sulle esigenze specifiche di quel territorio.

L'introduzione del federalismo si propone di responsabilizzare i governi decentrati che, dovendo farsi carico degli eventuali deficit $\mathrm{e}$ rispondere delle proprie scelte di fronte ai cittadini elettori, saranno stimolati ad assumere comportamenti più efficienti grazie al confronto diretto tra amministratori ed amministrati [2]. Tuttavia con buona pace dei padri del federalismo stesso, da Tocqueville a Cattaneo, in Italia la devoluzione dei poteri nasce prevalentemente come comportamento non derivante da una specifica e maturata progettualità di evoluzione istituzionale da parte della classe politica quanto da una sorta di reazione tattica al successo elettorale leghista della metà degli anni '90 e alla rincorsa di quella quota di elettorato [3].
*Università di Ferrara

§SIFEIT, Società Italiana per Studi di Economia ed Etica sul Farmaco e sugli Interventi Terapeutici 
La sanità diventa l'oggetto primario del cambiamento federalista non tanto perché esista una effettiva esigenza di salute così diversa tra regioni in termini di epidemiologia o morbilità, quanto perché le regioni, investite non a caso dai nuovi poteri di autonomia [4], hanno circa 1' $80 \%$ del loro bilancio destinato alla sanità, dovendo garantire il più elevato grado di assistenza ma facendo quadrare i conti delle risorse disponibili. Il primo grande e spinoso obbiettivo del federalismo diventa perciò proprio l'assistenza sanitaria.

\section{ALLE ORIGINI DEL FEDERALISMO}

Prima di affrontarne la componente legata alla sanità, vale la pena dare un'occhiata alle origini del federalismo. Il termine deriva dall'aggettivo francese fédéral, ma ha la sua radice etimologica nel vocabolo latino foedus, patto. Esso designa una dottrina e tendenza politica volta a organizzare lo Stato sulla base dei principi dell'autogoverno, dell'autonomia e del più largo decentramento amministrativo, in contrapposizione allo Stato unitario accentratore. Lo Stato federale così formato è composto da una pluralità di Stati membri, ognuno con ampia autonomia legislativa, fatta eccezione per quelle materie di rilevanza internazionale (politica estera, difesa, etc.) di esclusiva competenza dello Stato federale la cui legge, in caso di conflitto, prevale sulla legge dello Stato membro. In ciò sta la differenza fra gli Stati federali e le altre unioni interstatali più o meno strette e durature - quali le unioni reali monarchiche o le confederazioni di Stati - i cui membri mantengono sempre una loro individualità e capacità giuridica internazionale e, di riflesso, all'interno dell'unione una maggiore libertà d' azione. Generalmente in uno Stato federale il potere legislativo federale viene esercitato da un Parlamento bicamerale, variamente chiamato: una Camera bassa, composta di rappresentanti del popolo; una Camera alta, composta di rappresentanti dei singoli Stati membri.

Il federalismo trae la sua origine da abbozzi di pensiero dei secoli scorsi: la dottrina elaborata dal tedesco Althusius nella Politica methodice digesta (1603), così come quella di Montesquieu nello Spirito delle leggi (1748) e di Kant nel saggio Sulla pace perpetua (1795). Cambiano in tali autori i valori di riferimento alla base delle loro concezioni: nel primo è il rispetto del principio di consociazione, posto a fondamento della politica; nel secondo è la libertà intrinseca a un modello costituzionale alternativo al dispotismo; nel terzo è la pace perpetua, che solo una repubblica federale può salvaguardare.

Il moderno federalismo, però, è nato di fatto soltanto con Jefferson e la formazione degli U.S.A. e con il serrato dibattito ideologico a essa collegato, intrecciato dai pubblicisti poli- tici americani A. Hamilton, J. Jay, J. Madison, che nel 1788 diedero mano alla raccolta di saggi The Federalist, ossia il Commento alla Costituzione degli Stati Uniti d'America. Partirono da una premessa: ad essi non bastò più il Parlamento come organo di controllo unico del potere esecutivo e chiesero che lo Stato assumesse una struttura federale in quanto, solo in tal modo, il cittadino avrebbe goduto di sicure garanzie grazie alle quali la politica non avrebbe potuto limitare la sua autonomia. Il pensiero politico federalista nacque pertanto in polemica con la teoria dello Stato sovrano, accentrato e assoluto e come diretta filiazione delle correnti politiche liberali anglosassoni del XVII secolo. L'opera degli autori del Federalist fu davvero imponente per ampiezza e coerenza ed evitò la formazione di strapoteri volti a snaturare le comunità di base e il governo federale.

Un particolare significato del federalismo emerge dalle vicende della Rivoluzione francese, e soprattutto dal contrasto tra girondini e giacobini: i primi reclamavano una struttura federale della Francia, al posto del regime accentrato voluto dai giacobini. In tal senso, il federalismo accentuava il suo orientamento autonomista e cantonalista all'interno di uno stato nazionale di tradizione fortemente unitaria. Tale tendenza fu approfondita nell' $800 \mathrm{da}$ Proudhon in Francia, da Frantz in Germania, e da Cattaneo in Italia. Pur essendo Marx ed Engels contrari al federalismo, nel socialismo marxista della Seconda Internazionale vi fu una fioritura federalista con le opere di K. Renner e di O. Bauer per ciò che concerneva l'articolazione dell'impero asburgico e la prospettiva dell'unità europea. Di coscienza europea intesa come grande comunità spirituale da contrapporsi ai singoli Stati si può parlare fin dal XVIII secolo. Però, sebbene gli illuministi abbiano in buona sostanza sentito e, di conseguenza, abbiano cercato di rappresentare una grande società degli spiriti, un tipo di ampia "Repubblica europea dei letterati", soltanto alla fine del '700, con la Rivoluzione francese, un sentimento definibile di comunità continentale esplose e perciò, solo in seguito, pure l'azione politica fu talvolta tesa ad un fine sovranazionale e unitario.

In linea generale va ricordato che esistono diversi gradi di federalismo: gradi forti e gradi deboli. Fra i federalismi forti vanno citati gli Stati Uniti d'America, il Canada e gli altri grandi stati federali del centro e del Sud America, come il Brasile, l'Argentina, il Venezuela e il Messico. Pienamente federali sono l'Austria, la Svizzera e, dal 1992, il Belgio, unico esempio di abbinamento fra monarchia e struttura federale. Fra i federalismi deboli vanno indicati la Germania Federale e la Spagna, anche se i Lander e le Autonomie hanno comunque poteri assai più incisivi di quelli delle nostre re- 
gioni. Anche l'ex U.R.S.S., a suo modo, era uno stato federale, fino al suo scioglimento avvenuto nel 1991, mentre oggi la derivante Comunità degli Stati Indipendenti (C.S.I.) è confederale.

\section{IL FEDERALISMO SANITARIO}

$\mathrm{Ci}$ sono delle tappe normative che hanno segnato il processo di progressiva attribuzione delle competenze e delle responsabilità in ambito sanitario alle Regioni [5]:

- la Legge 502 del 1992: attribuisce alle regioni la responsabilità organizzativa dell'assistenza sanitaria;

- la modifica del titolo V della Costituzione alla fine del 2000, al termine della precedente legislatura (votata con soli quattro voti di maggioranza la variazione di cinque articoli e confermata con il referendum del 7 ottobre 2001 e la conseguente legge n. 3 del 18 ottobre 2001, che definisce la concorrenza legislativa tra Stato e Regioni in materia anche di sanità);

- il Dlgs 56/2000 sul federalismo fiscale, che prevede una ripartizione dei trasferimenti erariali alle regioni sulla base della compartecipazione all'IVA delle stesse: ogni regione si "riprende" risorse in base a quante ne ha versate. È previsto un fondo di perequazione che riequilibra le risorse indirizzate alle regioni ricche ed a quelle povere. La normativa prevedeva una decorrenza graduale e progressiva con uno status di pieno regime nel 2013;

- Legge 405 del 19.11.2001 (di conversione del Decreto 347) a recepimento dell'accordo Stato-Regioni dell'agosto 2001 sulla spesa sanitaria e sul trasferimento alle Regioni di rilevanti funzioni gestionali ed organizzative, tra questi l'accesso a risorse supplementari solo a Regioni finanziariamente virtuose;

- il DCPM 29/11/2001 sulla nuova definizione dei Livelli Essenziali di Assistenza (LEA) volti a mantenere una sostanziale omogeneità nella tipologia delle prestazioni sanitarie erogate dalle diverse Regioni;

- l'approvazione in prima lettura pochi mesi fa della riforma costituzionale che definisce una attribuzione non più concorrenziale delle competenze sanitarie tra stato e regioni. Esecutiva, previa conclusione dell'iter parlamentare e referendario, non prima del 2011.

Intrapresa quindi la strada federalista, ritenuta a ragione o a torto quella da seguire, la partita si gioca principalmente su terreni posti su livelli diversi ma contigui [6].
Uno è quello istituzionale-costituzionale e definisce la competenza e concorrenza legislativa, i termini di sussidiarietà tra stato ed enti locali e i relativi meccanismi di controllo e coordinamento. Ciò a valle della modifica del titolo V della Costituzione, ed in parte dell' art. 117 , che pone su un piano parallelo, perciò concorrente, e non più verticale, il potere centrale e quelli locali. Il punto chiave è nel limitare o escludere sovrapposizioni nella legiferazione tra stato ed enti locali e nel non dare luogo a conflittualità incrociate in una sorta di "sindacalismo" istituzionale. In merito, dal 2001 si sono moltiplicati i contenziosi alla Corte Costituzionale tra stato e regioni [7].

Un altro livello è quello equitativo e solidaristico, ovvero del mantenimento di una sostanziale omogeneità nella tipologia delle prestazioni sanitarie erogate dalle diverse Regioni e di conseguenza della re-distribuzione delle risorse finanziarie tra le regioni per garantire tale omogeneità [8]. Ad oggi le regioni si finanziano attraverso una sorta di trasferimento erariale da parte dello Stato centrale che viene ancora definito convenzionalmente fondo sanitario nazionale (FSN). L'entità delle risorse trasferite ad ogni regione è calcolata su criteri basati sulle quote capitarie, ovvero sul numero di abitanti indicizzato ad alcuni elementi di cui il prevalente è la presenza di anziani. Le risorse al fondo sanitario nazionale [9], benché centralizzato, arrivano tuttavia soprattutto da fonti locali (Tabella I). Già la legge 405/ 2001 prevedeva una sorta di meccanismo finanziariamente premiante con accesso a risorse centrali aggiuntive, per le regioni capaci di rimanere nei rispettivi budget sanitari. Meccanismo di identificazione del fabbisogno e conseguente riparto delle risorse reiterato anche di recente [10] nell'avvenuto accordo StatoRegioni (Tabella II). Sull'altro piatto della bilancia, quello delle prestazioni erogate, i LEA costituiscono lo strumento atto a garantire

\begin{tabular}{lc}
\multicolumn{1}{c}{ Fonte } & \% del finanziamento \\
\hline IRAP & 39,1 \\
Partecipazione Regioni Statuto Speciale & 6,2 \\
Addizionale IRPEF & 6,2 \\
Entrate regionali proprie & 2,9 \\
Accisa benzina & 0,1 \\
Fondo sanitario (solo Sicilia e Sardegna) & 3,7 \\
Compartecipazione all'IVA & 41,7 \\
Totale & 100,0 \\
\hline
\end{tabular}

Tabella I

Fonti di finanziamento della sanità pubblica (2003) [Fonte: Ministero dell'economia, Ragioneria generale dello Stato] 


\begin{tabular}{|c|c|c|}
\hline Regione & Fabbisogno & $\%$ \\
\hline Piemonte & 6.622 & 7,7 \\
\hline Valle d'Aosta & 183 & 0,21 \\
\hline Lombardia & 13.635 & 15,86 \\
\hline Bolzano & 664 & 0,77 \\
\hline Trento & 711 & 0,83 \\
\hline Veneto & 6.807 & 7,92 \\
\hline Friuli & 1.850 & 2,15 \\
\hline Liguria & 2.614 & 3,04 \\
\hline Emilia Romagna & 6.355 & 7,39 \\
\hline Toscana & 5.594 & 6,51 \\
\hline Umbria & 1.328 & 1,54 \\
\hline Marche & 2.303 & 2,68 \\
\hline Lazio & 7.764 & 9,03 \\
\hline Abruzzo & 1.948 & 2,27 \\
\hline Molise & 495 & 0,58 \\
\hline Campania & 7.962 & 9,26 \\
\hline Puglia & 5.753 & 6,69 \\
\hline Basilicata & 883 & 1,03 \\
\hline Calabria & 2.917 & 3,39 \\
\hline Sicilia & 7.217 & 8,39 \\
\hline Sardegna & 2.357 & 2,74 \\
\hline
\end{tabular}

Tabella II

Fabbisogno SSN 2005 [Fonte: Conf. Stato-Regioni 23/3/2005]

\begin{tabular}{|c|c|}
\hline Assistenza ospedaliera & $\begin{array}{l}\text { Incremento dell'appropriatezza e manovra sulle } \\
\text { strutture di offerta (4 posti letto per mille abitanti). } \\
\text { Altro obiettivo è quello della continuità } \\
\text { dell'assistenza che leghi l'ospedale al territorio }\end{array}$ \\
\hline Assistenza specialistica & $\begin{array}{l}\text { Ridefinizione e manutenzione del nomenclatore } \\
\text { tariffario, incremento del grado di appropriatezza } \\
\text { coniugato con gli adeguamenti alle innovazioni } \\
\text { scientifiche e tecnologiche e con metodologie di } \\
\text { controllo di gestione a livello distrettuale }\end{array}$ \\
\hline Assistenza farmaceutica & $\begin{array}{l}\text { Garanzia senza alcun ticket del rimborso dei } \\
\text { farmaci inclusi nella classe A. Facoltà di } \\
\text { modulazione della assistenza farmaceutica con } \\
\text { misure di copayement ed organizzative (es.: } \\
\text { distribuzione) in relazione all'andamento della } \\
\text { spesa. Totale carico per la fascia C }\end{array}$ \\
\hline $\begin{array}{l}\text { Area dell'integrazione } \\
\text { socio-sanitaria }\end{array}$ & $\begin{array}{l}\text { Utilizzo di Fondi Integrativi. Organizzazione della } \\
\text { rete delle strutture di offerta. Omogenea modalità } \\
\text { di rilevazione del bisogno e classificazione del } \\
\text { grado di non autosufficienza o dipendenza. } \\
\text { Prestazioni a favore delle fragilità sociali, owvero } \\
\text { minori, donne, famiglia, anziani, disabili, pazienti } \\
\text { psichiatrici, persone con dipendenza da alcool, } \\
\text { droghe e farmaci, malati terminali, persone con } \\
\text { patologie da Hiv }\end{array}$ \\
\hline
\end{tabular}

Tabella III

Principali criteri per la definizione dei Lea [Fonte: D.p.c.m. 29 novembre 2001 "Definizione dei livelli essenziali di assistenza" (mod.)] omogeneità di diritti sulla salute per tutti i cittadini italiani, a prescindere dal tipo di organizzazione sanitaria preposta. Sono citati per la prima volta dai decreti legislativi 502 e 517 del '93 e oggetto di una ridefinizione nella cosiddetta riforma "Bindi" (D.Lgs. 229/99) e hanno trovato una compiuta cornice legislativa nella stessa riforma costituzionale. Con il Dcpm del 29/ 11/2001 sui LEA si intende, nello spirito originale del legislatore, dare luogo ad un riferimento nazionale omogeneo per l'offerta di servizi sanitari, in termini sia quantitativi che qualitativi. Il documento sui LEA ha inteso rispondere a questo principio (Tabella III), individuando liste negative di prestazioni e percorsi terapeutici ottimali, anche attraverso la revisione degli attuali DRG, per l'assistenza ospedaliera e territoriale.

L'equilibrio tra rispetto per i LEA e risorse concordate e disponibili rappresenta l'elemento di spinta ad una maggiore efficienza tecnica e allocativa da parte di ogni singola Regione. I LEA incorporano praticamente tutte le prestazioni già precedentemente erogate dal SSN; è nella discrezionalità delle singole regioni modularne l'applicazione in modo efficiente e, almeno per quelle comprese negli allegati del relativo decreto (Tabelle IV-V), a seconda delle proprie disponibilità finanziarie e della propria volontà politica. I LEA rivedono il concetto di uniformità territoriale nell'erogazione delle prestazioni, con le possibili conseguenti implicazioni equitative che ne conseguono, aprendo così la strada alla formale ricerca di risorse aggiuntive in modo autonomo da parte di ogni singola Regione [11]. Da un paio di anni, infatti, stiamo assistendo all'aumento delle voci di finanziamento integrativo regionale: addizionale IRPEF, accisa sui carburanti, maggiorazione del bollo auto, IRAP, ticket, etc.

\section{L'EQUILIBRIO TRA RISORSE E LEA ATTRAVERSO L'EFFICIENZA DI DIFFERENTIMODELLIORGANIZZATIVI TRA REGIONI}

Il raggiungimento del miglior grado di efficienza tecnica e allocativa per il raggiungimento dell'equilibrio tra erogazione dei LEA e risorse disponibili passa attraverso la struttura organizzativa dei servizi [12]. Sono individuabili, di fondo, tre modelli principali di riferimento: quello basato sulla equiparazione competitiva degli erogatori (pubblici e privati/convenzionati) in cui la regione fa da arbitro-regolatore (ad esempio la Lombardia) che dovrebbe funzionare sulla concorrenza competitiva basata sulla efficacia ed efficienza (costi, prezzi, qualità); quello della programmazione negoziata e contrattata tra regione e proprie unità funzionali erogatrici ASL ed Aziende Ospedaliere (ad 
esempio l'Emilia e la Toscana) nel quale l'operatore privato accreditato può essere più o meno presente; quello a concorrenza passi$v a$ (diverse regioni meridionali), con quantità di prestazioni non predeterminata e ad esito aperto, e con conseguente gestione dei bilanci a piè di lista.

Nel modello "lombardo" di rapporto con gli erogatori privati di prestazioni sanitarie, al quale si è ispirata finora solo parzialmente qualche altra regione, il coinvolgimento di erogatori privati prevede parità competitiva con gli erogatori pubblici, con uguali tariffe e rientranti in un unico budget funzionale.

Il modello "programmatorio", adottato ad esempio da Emilia-Romagna, Toscana ed Umbria, prevede l'accreditamento del privato in una logica subordinata alla programmazione regionale, quindi alle esigenze specifiche nel rapporto domanda-offerta del SSN, ma su budget prefissati e con tariffe, per il privato, di solito inferiori a quelle adottate per le strutture pubbliche.

Il modello della concorrenza passiva appare, per ovvi motivi, il meno controllato soprattutto sotto il profilo dei consumi e della spesa, tendenti alla crescita incontrollata.

Va notato come le recenti decisioni delle regioni, in merito al rapporto con l'erogatore privato, rispetto al passato, tendano a convergere su posizioni più vicine e meno distanti tra loro, sia in chiave programmatoria che di controllo e verifica della spesa e della qualità delle prestazioni.

Il modello orientato sulla libera concorrenza è solitamente più difficile da far funzionare perché l'offerta privata fa lievitare domanda e consumi. Richiede quindi un contesto di regole e, soprattutto, controlli di comportamento. L'esempio del condizionamento distorsivo dei comportamenti nell'erogazione delle prestazioni dopo l'adozione dei DRG fa lezione in merito [13]. Inoltre il modello "lombardo" funziona a fronte di una programmazione che deve essere totalmente indipendente dall'offerta, escludendo quindi qualunque tipo di integrazione verticale tra programmazione della domanda ed offerta, pena una commistione tra chi paga e chi eroga, che pur potendo mantenere un buon grado di efficacia annulla il beneficio di efficienza.

Il modello della programmazione e condivisione delle responsabilità rappresenta il più tradizionale dei sistemi di Servizio Sanitario Nazionale, basati sul National Healthcare System britannico (NHS). Le varianti corrono sul gradiente di contrattazione e corresponsabilizzazione delle entità erogatrici delle prestazioni verso il principale gestore del budget (fundholder) che nel nostro sistema è di fatto rappresentato dagli assessorati regionali alla sanità. Si va, in sintesi dall'estremo della pro- a. Chirurgia estetica non conseguente a incidenti, malattie o malformazioni congenite

b. Circoncisione rituale maschile

c. Medicine non convenzionali (agopuntura - fatta eccezione per le indicazioni anestesiologiche-, fitoterapia, medicina antroposofica, medicina ayurvedica, omeopatia, chiropratica, osteopatia nonché tutte le altre non espressamente citate

d. Vaccinazioni non obbligatorie in occasione di soggiorno all'estero

e. Certificazioni mediche (con esclusione di quelle richieste dalle istituzioni scolastiche ai fini della pratica non agonistica per i propri alunni, ai sensi dell'art. 31 del d.p.r. 270/2000 e dell'art. 28 del d.p.r. 272/2000) non rispondenti a fini di tutela della salute collettiva, anche quando richieste da disposizioni di legge (incluse le certificazioni di idoneità alla pratica di attività sportiva, agonistica e non, idoneità fisica allimpiego, idoneità al servizio civile, idoneità all'affidamento e all'adozione, rilascio patente, porto d'armi, ecc.

f. Le seguenti prestazioni di medicina fisica, riabilitativa ambulatoriale: esercizio assistito in acqua, idromassoterapia, ginnastica vascolare in acqua, diatermia a onde corte e microonde, agopuntura con moxa revulsivante, ipertermia NAS, massoterapia distrettuale riflessogena, pressoterapia o pressodepressoterapia intermittente, elettroterapia antalgica, ultrasuonoterapia, trazione scheletrica, ionoforesi, laserterapia antalgica, mesoterapia, fotoforesi terapeutica, fotochemioterapia extracorporea, fotoforesi extracorporea. Su disposizione regionale la laserterapia antalgica, 1 'elettroterapia antalgica, l'ultrasuonoterapia e la mesoterapia possono essere incluse nella Lista delle prestazioni parzialmente escluse dai LEA

\section{Tabella IV}

Lista delle prestazioni totalmente escluse dai LEA [Fonte: D.p.c.m. 29 novembre 2001 "Definizione dei livelli essenziali di assistenza", allegato 2A]

a. Assistenza odontoiatrica: limitatamente alle fasce di utenti e alle condizioni indicate al comma 5 art. 9 del d.lgs. 502/1992 e successive modifiche e integrazioni

b. Densitometria ossea limitatamente alle condizioni per le quali vi sono evidenze di efficacia clinica

c. Medicina fisica, riabilitativa ambulatoriale: l'erogazione delle prestazioni ricomprese nella branca è condizionata alla sussistenza di taluni presupposti (quali la presenza di quadri patologici definiti, l'età degli assistiti, un congruo intervallo di tempo rispetto alla precedente erogazione, ecc.) owero a specifiche modalità di erogazione (ad es. durata minima della prestazione, non associazione con altre prestazioni definite, ecc.), fatto salvo quanto previsto alla Lista di prestazioni totalmente escluse dai LEA, punto f])

d. Chirurgia refrattiva con laser ad eccimeri erogabile limitatamente a casi particolari di pazienti con anisometropia grave o che non possono portare lenti a contatto o occhiali

Tabella V

Lista delle prestazioni parzialmente escluse dai LEA [Fonte: D.p.c.m. 29 novembre 2001 "Definizione dei livelli essenziali di assistenza", allegato 2B]

grammazione verticistica, imposta alle strutture, al modello di "quasi" mercato già proprio del NHS, in cui pur rimanendo all'interno del servizio pubblico c'è una sorta di mercato interno (internal market) tra i fornitori pubblici di servizi, con susseguente contrattualizzazione tra fundholder ed erogatore e relativa condivisione e distribuzione del rischio [14]. 


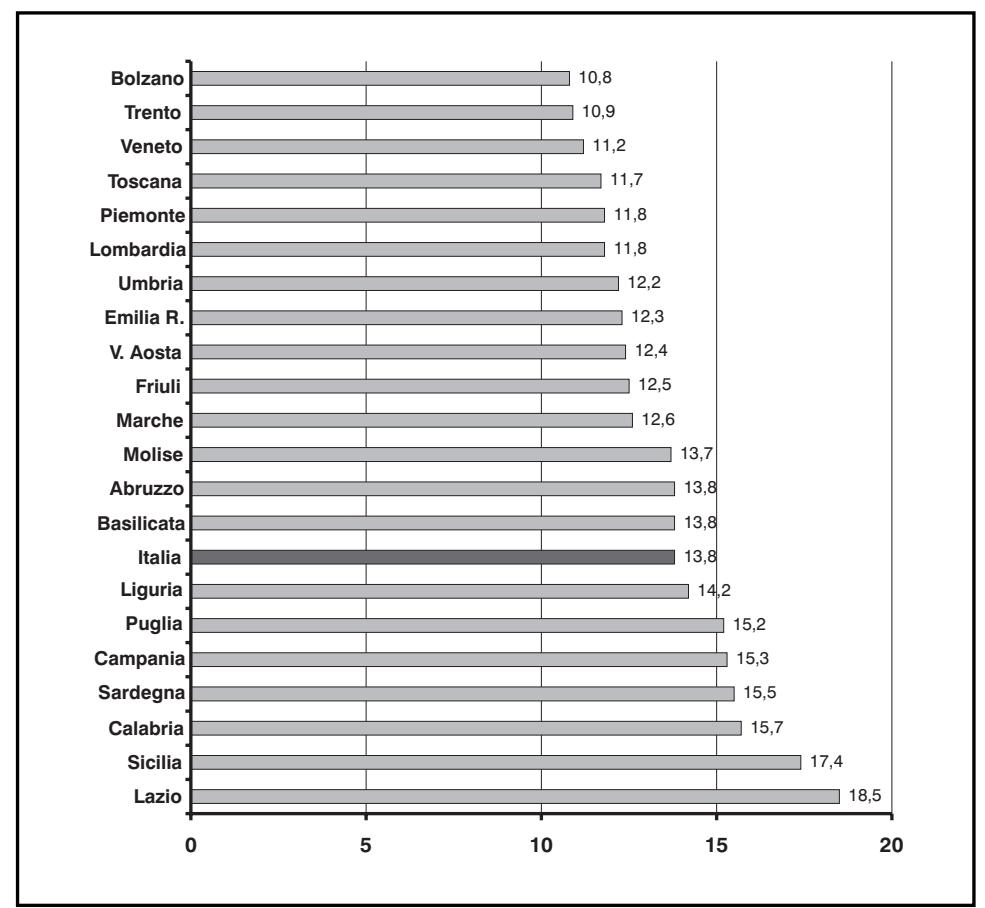

Figura 1

Percentuale di spesa farmaceutica pubblica su spesa SSN totale [Fonte: Cergas - Bocconi, su II Sole 24 Ore del 16/5/2005, pag. 23]

\section{SOSTENIBILITÀ FINANZIARIA E PROGRAMMAZIONE LOCALE BASATA SUL PROCESSO DI RILEVAZIONE DEI BISOGNI}

Il processo federalista si è sviluppato in modo molto accelerato e disordinato con soggetti, le regioni, chiamate a responsabilità, soprattutto finanziarie, senza avere di fatto completezza di strumenti normativi, fiscali e gestionali per assolverle. Sarebbe stato presumibilmente più funzionale creare prima l'infrastruttura istituzionale e legislativa di fondo per definire competenze e responsabilità rispettivamente tra stato, regioni ed altri enti locali, capace di garantire il necessario grado di sussidiarietà verticale tra istituzioni.

La sanità, pesando per oltre 1'80\% sui budget regionali è diventata forzosamente e all'improvviso il banco di prova del federalismo. Oltre a fiscalità fortemente differenziate tra regioni, la variabilità più elevata si è avuta sull'assistenza farmaceutica, fortemente penalizzata nei modi più vari, pur rappresentando solo un sesto della spesa sanitaria pubblica ma permanendo estremamente variabile tra le regioni, in alcune delle quali sotto scarso controllo (Figura 1).

La riforma costituzionale approvata in prima lettura, lungi dal divenire esecutiva, visto l'iter parlamentare e poi referendario ancora da seguire, paradossalmente sembra centralizzare alcune funzioni decisionali sulla salute, anziché decentrarle alle regioni alle quali competerebbe l'assistenza. Uno dei punti cruciali, tuttavia, è che non esiste ancora un indirizzo chiaro sul carattere solidaristico del federalismo fiscale, ovvero se e quanto regioni "ricche" debbano compensare finanziariamente regioni "povere".

Per ora, il faticoso abbandono del centralismo non ha ancora permesso di costruire un sistema alternativo caratterizzato da un buon livello di consenso culturale e politico e che abbia la concreta possibilità di raggiungere migliori risultati rispetto al passato sia sul piano degli output (la qualità organizzativa del sistema) sia su quello degli outcome (i risultati di salute), pur con tutta la difficoltà di rilevare questo dato in maniera da poterlo correlare con i servizi prestati. Il regionalismo, in teoria, pone alla propria base la possibilità che i sistemi di erogazione delle prestazioni siano strutturati in maniera diversa, richiesta che corrisponde alle più moderne teorie di governo dei sistemi complessi, le quali rifiutano la modellistica rigida e illuministica alla quale siamo abituati, per privilegiare sistemi non lineari, sensibili alla variabilità delle culture, delle professionalità, delle attese e delle speranze dei cittadini.

È però indispensabile immettere nel sistema federale alcune premesse di carattere fondamentalmente etico che, pur non interferendo con le decisioni delle singole regioni, garantiscano il cittadino su alcuni servizi irrinunciabili e su alcuni risultati di salute. Questo può rappresentare l'unica strada che - nel rispetto delle autonomie - garantisca sostanzialmente il cittadino di fronte a diversi sistemi sanitari. Il regionalismo sanitario trova risposte solo in una crescita civile che riconosca a tutti i cittadini di una determinata area diritti irrinunciabili, dai quali conseguono comportamenti anche visibilmente adeguati: l'attenzione deve essere rivolta a bisogni primari quali l'accoglienza, la dignità nei rapporti personali, il rispetto della sofferenza e delle pressioni psicologiche e fisiche che questa impone.

In termini di organizzazione e gestione l'incertezza si riflette sulla programmazione sanitaria, a tutti i livelli, primariamente regionale. I diversi modelli di organizzazione osservati nelle varie regioni, compresi negli estremi del modello lombardo da un lato e quello toscano-emiliano dall'altro, iniziano a convergere nella valorizzazione del processo programmatorio che tenga conto del doppio binomio offerta-risorse e domanda-bisogni, in un processo di pianificazione e monitoraggio dell'erogazione delle prestazioni che parta dal "basso verso l'alto" (bottomup) e sia capace di intercettare e rilevare specificamente i bisogni e la domanda sanitaria locale. In una prospettiva sempre più credibile di integrazione tra servizi sanitari e sociali e di conseguente spostamento sul territorio delle prestazioni, il ruolo degli enti locali, municipalità incluse, assume ulteriore centralità di indirizzo e contributo decisionale. 


\section{LA NECESSARIA MATURAZIONE DEL PROCESSO FEDERALISTA}

Si potrebbe essere preoccupati rispetto alla concreta prospettiva di sistemi regionali che tendono a differenziarsi, in termini di sostenibilità finanziaria, equità e socialità. Del resto la massa di innovazioni giuridiche e organizzative in corso cala su una realtà di SSN più nominale che sostanziale, plasmata da tempo su una pletora di modelli di sanità e su livelli di prestazioni, di fatto, estremamente diversificati. Finanziamento, regionalismo e politica potranno essere in sintonia se si perseguirà un sistema non guidato da una domanda che si auto-riproduce, si auto-regola e perde ogni contatto con il vero bisogno.

Prevale la sensazione che la responsabilizzazione locale sia un processo, volenti o nolenti, sostanzialmente irreversibile. Ci si chiede se non sia strutturale al sistema l'impossibilità di delineare un quadro evolutivo chiaro e se la scelta di imporre un certo ordine sia illusoria e sbagliata: nei sistemi sanitari forse il compito della politica è quello di "governare il disordine", rinunciando ad imporre modelli generali, ma limitandosi a dare alcuni confini ad un insieme di attività, ciascuna delle quali segue regole proprie, non sempre tra loro in accordo e non sempre rispondenti ai tradizionali modelli di razionalità. Sarebbe inutile fare una storia dei nostri servizi per la salute e metterne in evidenza la storica incapacità di evolvere secondo regole predeterminate. Non vi è stato in passato alcun legame tra investimenti e prodotto, tra spesa corrente e risultati, tra bisogno di salute e quantità di specifici servizi. Il regionalismo pone alla propria base la possibilità che i sistemi di erogazione delle prestazioni siano strutturati in maniera diversa, cosa che corrisponde alle più moderne teorie di governo dei sistemi complessi, le quali rifiutano la modellistica rigida e illuministica per privilegiare i sistemi non lineari, sensibili alla variabilità delle culture, delle professionalità, delle attese e delle speranze dei cittadini.

Tuttavia deve permanere intangibile il criterio che nel sistema federalistico siano ferme alcune premesse di carattere fondamentalmente etico che garantiscano il cittadino su alcuni servizi irrinunciabili e su alcuni risultati di salute. Se la comunità, come mix tra enti locali e aggregazioni spontanee, interpreta le soggettività di un territorio con forti differenze concrete sul piano geografico ed antropologico, può svolgere un ruolo partecipativo nella programmazione, gestione e valutazione dell'assistenza. Questo permette anche di sfuggire a tentazioni di rigidità programmatorie estreme che troppo spesso rischiano di fallire in quanto viziate dall'errore di voler imporre un "ordine" dove invece la funzione di guida deve limitarsi ad aiutare la crescita di chi localmente esprime idealità, volontà e disponibilità di servizio in ambito sanitario.

Comunque, guardando a quei Paesi che già hanno adottato assetti federalisti [16], ed osservando in particolare la gestione della materia sanitaria, ad esempio in Canada, si evince come sia stato necessario un congruo numero di anni, di modifiche correttive e di passaggi conflittuali anche aspri per trovare l'assetto relativamente migliore. È quindi opportuno considerare la necessità di procedere con gradualità in un processo che richiede comunque tempi medio lunghi nei quali attraverso un monitoraggio attento ed oggettivo, dovranno essere via via apportate modifiche e cambiamenti progressivamente migliorativi.

\section{CONCLUSIONI}

Assorbendo gran parte delle risorse economiche regionali, la sanità costituisce di fatto il vero banco di prova del federalismo. La riduzione della distanze tra cittadini-elettori e propri amministratori intende responsabilizzare maggiormente questi ultimi ad una gestione della sanità più efficace ed efficiente. La storia del federalismo nei paesi civilmente più avanzati vede nell'equilibrio tra il potere centrale e i poteri locali l'elemento più cruciale, tema oggi ancora irrisolto in Italia.

Maggiori responsabilità decisionali decentrate possono dare il vantaggio di adattare meglio l'offerta di servizi alla specifica domanda locale, anche grazie all'applicazione di modelli organizzativi diversi, sia in termini programmatori che di rapporto tra erogazione dei servizi pubblici e privati.

La principale criticità di tale decentramento consiste però nel mantenimento di una sostanziale uniformità nella tipologia delle prestazioni sanitarie erogate dalle diverse Regioni e di conseguenza nella re-distribuzione delle risorse finanziarie tra le regioni per garantire tale omogeneità. È lo sforzo comune che oggi ruota intorno ai LEA e agli accordi tra Stato e regioni.

Ma nella disparità geografico-economica tipica del nostro Paese, per i servizi sanitari è particolarmente difficile, se non gradualmente e nel lungo periodo, conciliare socialmente la difforme autonomia finanziaria delle diverse regioni con lo storico carattere nazionale del sistema, che si basa su radicate motivazioni di natura etica e distributiva. Pur in un generale contesto di crescente individualismo e di convivenza civica tendenzialmente declinante, 1' "istituzione sanità" resta un caposaldo condiviso di appartenenza e perciò di sicurezza sociale, specialmente in questi tempi di incertezza generalizzata e con una società come la nostra progressivamente più anziana e perciò fragile.

Ciò rende non solo insopprimibile il ruolo di garanzia del livello nazionale ma, in tale ottica, ne reclama anzi un rafforzamento, soprat- 
tutto nel suo livello superiore di intervento rispetto alle competenze locali, sovrapponendosi ad esse quando occorra correggere le non infrequenti inefficienze, dispersioni e distorsioni esistenti sul territorio. Che proprio dal livello locale spesso sono in vario modo alimentate, con un risultato quindi paradossalmente in esatta antitesi proprio con i virtuosi princìpi ispiratori del federalismo stesso.

\section{BIBLIOGRAFIA}

1. Gianfrate F., Economia del settore farmaceutico, ed. Il Mulino, Bologna, 2004, pag. 19-20

2. Malandrino C., Federalismo. Storia, idee, modelli, ed. Carocci, Roma, 1998, pag. 170

3. Gianfrate F., Federalismo Sanitario, La Repubblica 4 aprile 2002, Roma, in "Salute", 2002, pag. 4-7

4. Fiorentini G., Interventi micro e riforme macro nel Servizio Sanitario Nazionale, in: I servizi sanitari in Italia, ed. Il Mulino, Bologna, 2004, pag. 9-41

5. G. U. della Repubblica Italiana, anno 2002 n. 33, 8 febbraio 2002

6. Gianfrate F., Analisi del federalismo sanitario e farmaceutico in Italia, in Financial Times Global Pharmaceutical Conference Proceedings, 6-7/11/2002, Londra, 2002, pag. 55-71

7. Rotelli E., L'eclissi del federalismo, ed. Il Mulino, 2003, Bologna

8. Collicelli C., Di Francia C., Regionalismo e Sanità, in Tendenze Nuove n. 1/2001, ed. Il Mulino, 2001, pag. 71-83

9. Ministero dell' Economia , Relazione Generale sulla situazione economica del Paese, 2004

10. Conferenza Stato Regioni, Presidenza del Consiglio dei Ministri, Rep. 2276 del 23 marzo 2005

11. Arcà S. , I livelli essenziali di assistenza, in Tendenze Nuove n. 4-5/2003, ed. Il Mulino, 2003, pag. 355-373

12. France G. , I Livelli Essenziali di Assistenza, in: G. Fiorentini (a cura di): I servizi sanitari in Italia, ed. Il Mulino, Bologna, 2003, pag. 101-102

13. Brenna A., Manuale di economia sanitaria, CIS editore, Milano, 1999, pag. 393-398

14. Mapelli V., Il sistema sanitario italiano, ed. Il Mulino, Bologna, 1999, pag. 122-126

15. Cergas-Bocconi, “Il trend delle regioni”, su Il Sole 24 Ore del 16 maggio 2005, pag. 23

16. Arachi G., I difficili equilibri del federalismo sanitario, Keiron, 1999, 2: 134-145 